REVISTA CIENCIAS BIOMÉ DICAS

PRESENTACIÓN DE CASOS CLÍNICOS

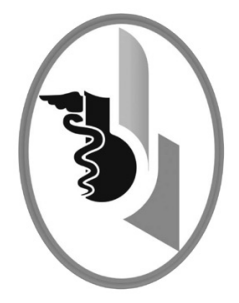

\title{
VASOESPASMO CEREBRAL SEVERO POR APOPLEJÍA PITUITARIA. REPORTE DE UN CASO Y REVISIÓN DE LA LITERATURA
}

\author{
SEVERE CEREBRAL VASOSPASM BY PITUITARY APLOPEXY. \\ A CASE REPORT AND LITERATURE REVIEW
}

Piña-Cabrales Sandra Milena ${ }^{1}$

Remolina-López Anderson Julián²

González-López Gilberto²

Ruiz-Cáez Karina María ${ }^{3}$

Correspondencia: kcaez05@hotmail.com

Recibido para evaluación: febrero - 12 - 2016. Aceptado para publicación: septiembre - 14- 2016

\section{RESUMEN}

Introducción: la apoplejía pituitaria (AP) es un síndrome caracterizado por infarto o hemorragia de la glándula hipófisis. Sus síntomas clínicos son caracterizados por dolor de cabeza súbito, náuseas, vómito, síntomas visuales y disfunción hormonal.

Caso clínico: mujer de 44 años quien presentó infarto cerebral secundario a AP. Se realizó resonancia magnética nuclear, panangiografía cerebral, tomografía axial computarizada y estudios hormonales, que permitieron el diagnóstico. Se realizó descompresión transesfenoidal urgente, aunque el desenlace fue fatal.

Conclusión: la isquemia cerebral secundaria por vasoespasmo cerebral es una complicación rara y potencialmente mortal de la AP. Rev.cienc.biomed. 2016;7(2):310-315.

\section{PALABRAS CLAVE}

Neurocirugía; Apoplejía pituitaria; Infarto cerebral.

\section{SUMMARY}

Introduction: pituitary apoplexy is a syndrome characterized by a stroke or bleeding from the pituitary gland. Its clinical symptoms are characterized by sudden headache, nausea, vomiting, visual impairment and hormonal dysfunction.

Case report: the goal of this article is to illustrate an unusual case of a 44 year -old woman who presented a secondary cerebral infarct by pituitary apoplexy. It was carried

1 Médico. Especialista en Neurocirugía. Fundación Centro Colombiano de Epilepsia y Enfermedades Neurológicas - FIRE. Cartagena. Colombia.

2 Médico. Estudiante de Postgrado. Neurocirugía. Facultad de Medicina. Universidad de Cartagena. Colombia.

3 Médico. Estudiante de Postgrado. Patología. Facultad de Medicina. Universidad de Cartagena. Colombia. 
out an urgency transphenoidal decompression surgery with fatal outcome.

Conclusion: secondary cerebral ischemia by cerebral vasospasm is a rare and potentially fatal complication of pituitary apoplexy. Rev.cienc.biomed. 2016;7(2):310-315.

\section{KEYWORDS}

Neurosurgery; Pituitary apoplexy; Cerebral infarction.

\section{INTRODUCCIÓN}

La apoplejía pituitaria (AP) es un síndrome clínico raro y potencialmente mortal, de instauración aguda, causado por el rápido crecimiento de un adenoma de hipófisis secundaria por infarto o hemorragia en la mayoría de los casos; caracterizado por el inicio súbito de cefalea, vómito, compromiso visual, oftalmoplejía y algunas veces alteración del estado de conciencia (1-3).

La AP ocurre en $0.6-10 \%$ de los pacientes con adenomas de hipófisis, sin embargo, el infarto cerebral como complicación es todavía más raro (1). La AP es más frecuente en adenomas no funcionantes, siendo en ocasiones la primera manifestación clínica. Entre los pacientes que se presentan con accidentes isquémicos transitorios (AIT) o eventos cerebrovasculares, los tumores hipofisiarios generalmente no se consideran entre los diagnósticos diferenciales, pero la obstrucción de las arterias carótidas internas por compresión directa del vaso a causa del aumento del volumen tumoral - el vasoespasmo pueden ocasionar infarto cerebral, en muchos de los casos potencialmente mortal $(1,4)$.

\section{CASO CLÍNICO}

Mujer de 44 años de edad sin antecedentes previos conocidos, excepto hipertensión arterial mal controlada, quien presentó cefalea intensa, náuseas, vómitos y posterior deterioro del estado de consciencia que la llevó rápidamente al coma. Razón por la cual necesitó intubación orotraqueal, soporte ventilatorio y manejo en la unidad de cuidados intensivos. En el examen físico se encontró crecimiento acral marcado, así como macroglosia y engrosamiento en los tejidos blandos de naríz, labios y orejas que sugerían fenotipo acromegálico.
Se realizó tomografía axial computarizada (TAC) cerebral simple que indicó lesión hiperdensa selar redonda con extensión supraselar, con sangrado subaracnoideo en cisternas de la base y edema cerebral secundario (Figura N01). La panangiografía cerebral (PAC) demostró severo vasoespasmo de las arterias cerebrales anteriores y media bilateral, descartándose lesión aneurismática de la circulación anterior (Figura No 2). La resonancia magnética nuclear (RMN) identificó tumor de densidad heterogénea selar compatible con adenoma grado IV B de Hardy, asi como hemorragia intratumoral (Figura No 3).

La paciente fue llevada a cirugía por vía tranesfenoidal, en la que se encontró

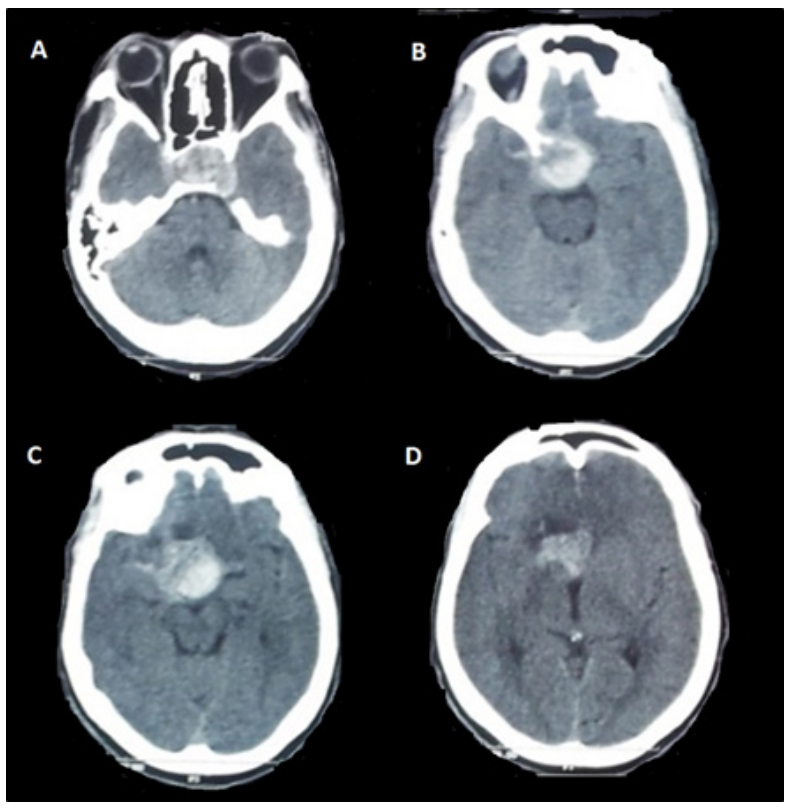

Figura No 1. TAC de cráneo simple en cortes axiales. A y $B$ a nivel de la base de cráneo, lesión hiperdensa nodular que ocupa la región selar con hipodensidades en su interior.

C: corte a nivel de la región mesencefálica, invasión a fosa anterior y fosa interpeduncular.

D: cortes a nivel del tercer ventrículo, colapso parcial de las astas frontales de los ventrículos laterales. 


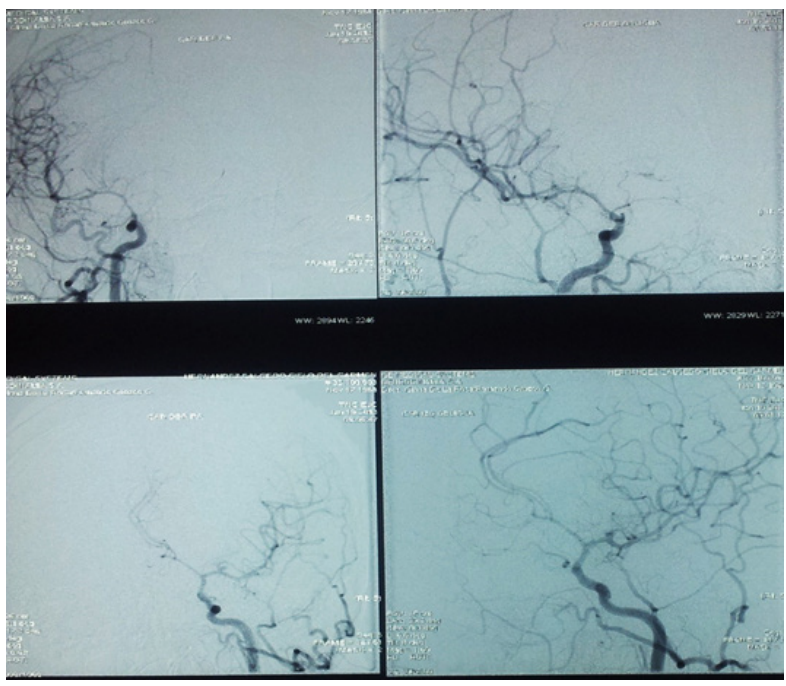

Figura No 2. Panangiografía cerebral. Severo vasoespasmo generalizado, predominio bilateral en la circulación anterior.

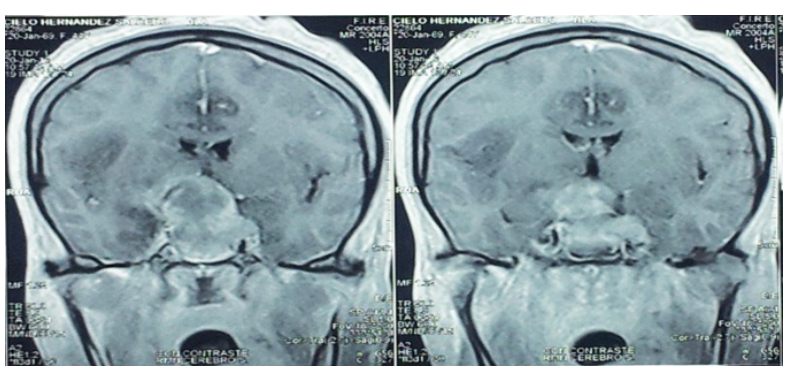

Figura No 3. RMN cerebral con gadolineo. Cortes coronales. Macroadenoma hipofisiario que capta heterogéneamente el medio de contraste. Grado IV-B de la clasificación de Hardy. Evaluación preoperatoria.

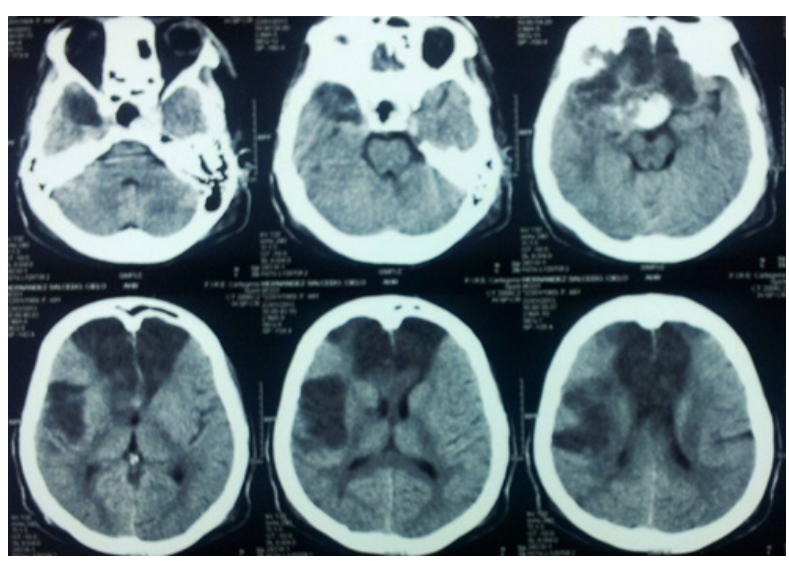

Figura No 4. TAC cerebral simple. Zonas hipodensas a nivel bifrontal y temporal derecha, compatibles con infarto de arteria cerebral anterior y cerebral media derecha. Evaluación posoperatoria. tumoración friable con sangrado reciente, que fue aspirado bajo visión microscópica hasta observar descenso del diafragma selar.

Durante el postoperatorio la paciente tuvo evolución tórpida, con inestabilidad hemodinámica y sin recuperación del estado de consciencia, por lo cual se solicitó TAC cerebral al segundo día posterior a la cirugía (Figura No 4). Se observaron grandes áreas de infarto en los territorios cerebrales anteriores y de la cerebral media derecha. La paciente continuó con deterioro sistémico y neurológico que finalmente conllevó a su deceso el quinto día posterior a la intervención. La patología confirmó el diagnóstico de adenoma hipofisiario con datos de apoplejía.

\section{DISCUSIÓN}

La AP es un síndrome de inicio súbito que se manifiesta con cefalea, vómitos, compromiso visual (hemianopsia bitemporal) y posterior disminución de la consciencia en el $75 \%$ de los casos. Cuando hay invasión del seno cavernoso puede ocurrir compromiso del tercer, cuarto o sexto par craneal, así como disminución en la sensibilidad facial y del reflejo corneal, sobre todo unilateral por compromiso del quinto par $(7,8)$. Es infrecuente el infarto cerebral secundario $(9,10)$.

La hemorragia intratumoral y el infarto hemorrágico en tumores de hipófisis se conocen como las principales causas de AP, las cuales son el resultado del rápido crecimiento tumoral del macroadenoma, lo que sobrepasa la suplencia sanguínea, resultando en necrosis isquémica y hemorrágica (2). El crecimiento tumoral hace que las arterias trabeculares que emergen de la arteria hipofisaria superior se compriman contra el diafragma selar produciendo isquemia y necrosis. En ocasiones, la arteria hipofisaria inferior irriga el adenoma, por lo que la isquemia puede iniciar en la glándula normal más que en la misma tumoración. Se ha señalado que la vasculopatía intrínseca es factor que hace más susceptible a los adenomas a infarto o hemorragia (11). Es así como la diabetes y la hipertensión arterial predisponen a apoplejía por degeneración en la microvasculatura, siendo peor en cetoacidosis diabética e hipertensión arterial maligna (9). 
ISSN: 2215-7840, 7(2), julio-diciembre 2016, Piña-Cabrales Sandra Milena, Remolina-López Anderson Julián, González-López Gilberto,

Ruiz-Cáez Karina María

Histológicamente no se han descrito factores intrínsecos a la apoplejía $(1,6)$. Los factores precipitantes se identifican en el $20-40 \%$ de los eventos. Se destacan la hipertensión arterial, irradiación, trauma menor de cráneo, terapia hormonal, embarazo, diabetes mellitus, procedimientos angiográficos, uso de medicación anticoagulante, diátesis hemorrágica, realización de estudios dinámicos de hipófisis ( $\mathrm{CRH}$, test de tolerancia a la insulina, TRH o GHRH), medicamentos como los agonistas dopaminérgicos, altas dosis de estrógenos, hemodiálisis y varios tipos de cirugía, incluyendo laminectomía lumbar y cirugía cardíaca $(2,4)$.

Bailey en 1898 fue el primero en describir la AP como una hemorragia catastrófica en un adenoma (8). Posteriormente, Dingley y Brougham asociaron la apoplejía con muerte súbita en pacientes con adenoma hipofisario. La incidencia de adenomas está entre el $2-12 \%$, siendo la AP en el $80 \%$ de los casos la primera manifestación del tumor pituitario no funcionante. La AP puede ocurrir a cualquier edad con predominio en la quinta y sexta década, siendo en la mayoría de las series en pacientes masculinos (3-5).

Se presentan predominantemente en macroadenomas no funcionantes, seguidos de los prolactinomas o secretores de hormona del crecimiento $(2,11,12)$, aunque en estos últimos se pudiese esperar la complicación por el compromiso de la microvasculatura del paciente acromegálico.

Los macroadenomas hipofisisarios con apoplejía causantes de isquemia cerebral son el resultado del rápido incremento del contenido intraselar con el consecuente incremento de la presión intraselar por encima de $47 \mathrm{mmHg}$, considerándose normal una presión menor a la presión intracraneal, 7-15 $\mathrm{mmHg}$. El aumento de la presión intraselar es suficiente para ocasionar invasión del seno cavernoso y estrechamiento de las arterias carótidas internas y sus ramas. Por otro lado, el vasoespasmo cerebral producido por la presencia de sangre en el espacio subaracnoideo causa irritación meníngea en la mayoría de los casos, generando estrechez de los vasos arteriales secundarios a meningitis química por la liberación al espacio subaracnoideo de sustancias vasoactivas contenidas dentro del tumor $(1,5,6)$.

Debido a lo inusual del infarto cerebral secundario por $A P$, se debe poner mucha atención en el diagnóstico diferencial y manejo oportuno, ya que en caso de infarto cerebral, lo más frecuente es que este sea isquémico por trombosis o embolia, lo cual requiere terapia antiplaquetaria y en algunos casos trombólisis. En caso de infarto isquémico por $\mathrm{AP}$, el tratamiento provocaría hemorragia severa en el tejido lesionado empeorando la sintomatología, por tanto, se hace necesario la realización de resonancia cerebral en forma urgente en caso de sospecha de esta entidad (1).

EI TAC es útil cuando no se dispone de RMN, permitiendo diagnóstico rápido. En fase aguda el TAC es capaz de identificar un tumor hipofisario en el 93-94\% de los casos, pero la hemorragia tumoral solo se precisa en el 21$46 \%$ de los pacientes. Suele demostrar áreas hiperdensas en el seno del tumor, cuando se trata de sangrado reciente o hipodensidad en la silla turca con realce en anillo de la lesión. la RMN sigue siendo el gold estándar de apoplejía, pues es capaz de demostrar si hay hemorragia, infarto, extensión supraselar, compresión del quiasma óptico o extensión al seno cavernoso (12).

En ocasiones es necesaria la realización de angiografía debido a la asociación de aneurismas intracraneales con adenomas de hipófisis, que tienen incidencia del $7.4 \%$, principalmente en los secretores de hormona del crecimiento (13).

Es importante la determinación de las concentraciones plasmáticas de TSH, $\mathrm{T} 4 \mathrm{~L}$, cortisol y prolactina, para valorar la necesidad de terapia hormonal sustitutiva y establecer la funcionalidad de los adenomas hipofisarios. Se debe realizar campimetría por confrontación o computarizada, para detectar defectos como hemianopsia o cuadrantanopsia bitemporal o pérdida de visión monocular (11).

Al realizar búsqueda bibliográfica del reporte de casos de tumor hipofisiario y stroke o adenoma e isquemia cerebral, desde 1952 hasta la fecha, se encontraron trece informes 
de isquemia cerebral debida a vasoespasmo secundario por hemorragia subaranoidea causada por AP. Diez correspondían a hombres con edades comprendidas entre 1555 años $(3,6,14-22)$, al parecer este sería el tercer caso reportado en mujeres.

Los pacientes con AP deben ingresar de forma urgente para estabilización, evaluación clínica, hormonal y radiológica, así como para tratamiento médico o quirúrgico. La insuficiencia suprarrenal aguda secundaria se observa en aproximadamente dos tercios de los pacientes con apoplejía. La hipocortisolemia hace que la vasculatura sea menos sensible a los efectos presores de las catecolaminas, con inestabilidad hemodinámica resultante, por esto se debe iniciar reposición intravenosa antes de verse instauradas las complicaciones. Es útil la hidrocortisona $100-200 \mathrm{mg}$ en bolo y luego 2-4 mg por hora en infusión continua por seis horas o 50-100 mg cada seis horas por vía IM. Una vez superado, la hidrocortisona debe ser rápidamente disminuida a dosis de 20 a $30 \mathrm{mg}$ por día, dividida en tres dosis (9).

Los estudios sobre descompresión temprana sugieren que no hay diferencia en el resultado visual y endocrinológico dependiendo del manejo conservador o quirúrgico $(1,2,6,9)$. Las guías actuales de la UK para el manejo de la AP recomiendan descompresión quirúrgica en casos de síntomas neuro-oftalmológicos o disminución del estado de consciencia. En algunos casos esta descompresión se considera urgencia neuroquirúgica, sin embargo, se ha encontrado que los resultados dentro de los primeros tres días o la primera semana son iguales, pero luego de una semana el pronóstico visual es menor. El $86 \%$ de los desórdenes visuales mejoran o resuelven si se operan dentro de los primeros ocho días versus $46 \%$ entre 9-34 días $(6,9)$.

Por lo tanto, la cirugía transesfenoidal semielectiva debe ser considerada para pacientes que están clínicamente estables pero no muestran deterioro en los signos neurooftalmológicos $(6,9,12)$.

La cirugía tranesfenoidal es de elección para los microadenomas y para la mayoría de los macroadenomas como en el caso presentado, dejándose la craneotomía para los inaccesibles. como aquellos con extensión supraselar, paraselar a lóbulos frontal y temporal (7).

Ante la evidencia clínica e imagenológica de vasoespasmo se recomienda el manejo con nimodipina y terapia hipertensiva e hipervolémica, similar al realizado en hemorragia subaracnoidea. La terapia hipertensiva se alcanza con una infusión de dopamina y noradrenalina, mientras que la hipervolémica es instituida con 3.5 litros/ día, incluso se sugiere tratamiento agresivo intervencionista para prevenir complicaciones isquémicas en el postoperatorio. El seguimiento imagenológico durante el POP de paciente de alto riesgo con apoplejía permite el reconocimiento temprano del vasoespasmo y su tratamiento oportuno $(1,11)$.

En el caso de la paciente presentada es probable que los cambios sucedidos rápidamente con el vasoespasmo ocurrieron en relación con la ya establecida vasculopatía previa dada por la HTA crónica mal controlada en relación con su acromegalia.

En general, se recomienda el seguimiento hormonal a las 4-8 semanas, y estudio de RMN a los tres y seis meses por cinco años (9). La recuperación parcial o completa de la función pituitaria se logra hasta en el $50 \%$ de los pacientes, principalmente en pacientes operados, aunque hasta el $80 \%$ requieren reposición hormonal posterior a la apoplejía (60-80\% reposición de corticoesteroides, $50-60 \%$ de hormona tiroidea, $10-25 \%$ desmopresina, 60-80\% testosterona). La diabetes insípida suele ser transitoria en el $5 \%$ y permanente en el $8 \%$ de los casos (9).

Hay series donde no hubo diferencias en la prevalencia del hipopituitarismo entre los manejados conservadora o quirúrgicamente. La mejoría en la agudeza visual se presenta en el $76 \%$, en el campo visual en el $79 \%$ y de la paresia ocular hasta en el $91 \%$, independiente del manejo (7). Los defectos en el campo visual no mejoran en pacientes con enfermedades asociadas y el $75 \%$ quedan con déficit visual permanente y atrofia óptica por isquemia prolongada (23).

En general el pronóstico en los adenomas de hipófisis con AP es favorable en el $60 \%$, el $19 \%$ 
ISSN: 2215-7840, 7(2), julio-diciembre 2016, Piña-Cabrales Sandra Milena, Remolina-López Anderson Julián, González-López Gilberto, Ruiz-Cáez Karina María

queda con síntomas menores, el 6\% con severa discapacidad y el $5 \%$ puede morir (11).

\section{CONCLUSIÓN}

Aunque infrecuente, es necesario tener en cuenta la AP como causa de isquemia cerebral y más cuando se tienen factores de riesgo establecidos. El abordaje de estas lesiones por vía transesfenoidal continua vigente como tratamiento de elección en caso de AP, principalmente cuando se cuenta con síntomas visuales marcados o alteración de la conciencia.

CONFLICTO DE INTERESES: ninguno que declarar.

FINANCIACIÓN: recursos propios de los autores. Estudios de laboratorio, insumos hospitalarios, medicamentos y honorarios profesionales fueron aportados dentro de la atención asistencial.

\section{REFERENCIAS BIBLIOGRÁFICAS}

1. Zhang C. et al. Cerebral infarction caused by pituitary apoplexy: case report and rewiew of literature. Turk Neurosurg. 2014;24(5):782-787.

2. Briet C, Salenave S, Chanson P. Pituitary apoplexy. Endocrinol Metab Clin North Am. 2015;44(1):199-209.

3. Ahmed SK, Semple PL. Cerebral ischaemia in pituitary apoplexy. Acta Neurochir (Wien). 2008;150(11):1193-6.

4. Chokyu I, Tsuyuguchi N, Goto T, Chokyu K, Chokyu M, Ohata K. Pituitary apoplexy causing internal carotid artery occlusion--case report. Neurol Med Chir (Tokyo). 2011;51(1):48-51.

5. Das NK, Behari S, Banerji D. Pituitary apoplexy associated with acute cerebral infarct. J Clin Neurosci. 2008;15(12):1418-20.

6. Rey-Dios R, Payner TD, Cohen-Gadol AA. Pituitary macroadenoma causing symptomatic internal carotid artery compression: surgical treatment through transsphenoidal tumor resection. J Clin Neurosci. 2014;21(4):541-6.

7. Elsässer Imboden PN et al. Apoplexy in pituitary macroadenoma: eight patients presenting in 12 months. Medicine (Baltimore). 2005;84(3):188-96.

8. Rajasekaran $\mathrm{S}$, et al. UK guidelines for the management of pituitary apoplexy. Pituitary Apoplexy Guidelines Development Group. Clinical Endocrinology. 2011;74:9-20.

9. Biousse V, Newman NJ, Oyesiku NM. Precipitating factors in pituitary apoplexy. J Neurol Neurosurg Psychiatry. 2001;71(4):542-5.

10. Ayuk J, McGregor EJ, Mitchell RD, Gittoes NJ. Acute management of pituitary apoplexysurgery or conservative management? Clin Endocrinol (Oxf). 2004;61(6):747-52.

11. Semple PL, Webb MK, de Villiers JC, Laws ER Jr. Pituitary apoplexy. Neurosurgery. 2005;56(1):65-72.

12. Semple $P L$, Jane JA, Laws ER. Clinical relevance of precipitating factors in pituitary apoplexy. Neurosurgery. 2007;61:956-962.

13. Sade B, Morh G, Tanpieri D, Rizzo A: Intrasellar aneurysm and a growth hormone-secreting pituitary macroadenoma. J Neurosurg. 2004;100:557-59.

14. Cardoso ER, Peterson EW. Pituitary apoplexy and vasospasm. Surg Neurol. 1983;20(5):391-5.

15. Pozzati E, Frank G, Nasi MT, Giuliani G. Pituitary apoplexy, bilateral carotid vasospasm and cerebral infarction in a 15-year-old boy. Neurosurgery. 1987;20(1):56-9.

16. Itoyama Y, Goto S, Miura M, Kuratsu J, Ushio Y, Matsumoto T. Intracranial arterial vasospasm associated with pituitary apoplexy after head trauma--case report. Neurol Med Chir (Tokyo). $1990 ; 30(5): 350-3$.

17. Sanno N, Ishii Y, Sugiyama M, Takagi R, Node Y, Teramoto A. Subarachnoid haemorrhage and vasospasm due to pituitary apoplexy after pituitary function tests. Acta Neurochir (Wien). 1999;141(9):1009-10.

18. Rodier G, Mootien Y, Battaglia F, Martinet O, Cohen E. Bilateral stroke secondary to pituitary apoplexy. J Neurol. 2003;250(4):494-5.

19. Akutsu H, Noguchi S, Tsunoda T, Sasaki M, Matsumura A. Cerebral infarction following pituitary apoplexy-case report. Neurol Med Chir (Tokyo). 2004;44(9):479-83.

20. Bhansali A, Dutta P, Khandelwal N, Pathak A, Vashisht R. Pituitary apoplexy: an unusual cause of frontal lobe syndrome. Australasian Radiol. 2005;49(2):127-3.

21. Jeon BC, Park YS, Oh HS, Kim YS, Chun BK. Pituitary apoplexy complicated by chemical meningitis and cerebral infarction. J Korean Med Sci. 2007;22(6):1085-9.

22. Radhiana H, Syazarina SO, Shahizon Azura AM, Azizi AB. Pituitary apoplexy: a rare cause of middle cerebral artery infarction. Med J Malaysia. 2013;68(3):264-6.

23. San Laureano FC, Villarejo IG, y cols. Apoplejía hipofisaria. Protocolo de actuación diagnóstica y terapéutica en urgencias. Emergencias. 2001; 13:279-282. 